\title{
Risk Factors and Prognosis in Patients with COVID-19 and Liver Injury: A Retrospective Analysis
}

This article was published in the following Dove Press journal: Journal of Multidisciplinary Healthcare

\author{
Jia-Xin Shen' \\ Ze-Hao Zhuang' \\ Qiao-Xian Zhang ${ }^{2}$ \\ Jiao-Feng Huang ${ }^{3}$ \\ Gong-Ping Chen ${ }^{4}$ \\ Ying-Ying Fang ${ }^{5}$ \\ Ai-Guo Cheng ${ }^{6}$
}

'Endoscopy Center, First Affiliated Hospital of Fujian Medical University, Fuzhou, People's Republic of China; ${ }^{2}$ Nursing Department, First Affiliated Hospital of Fujian Medical University, Fuzhou, People's Republic of China; ${ }^{3}$ Department of Liver Research Center, First Affiliated Hospital of Fujian Medical University, Fuzhou, People's Republic of China; ${ }^{4}$ Department of Pulmonary and Critical Care Medicine, First Affiliated Hospital of Fujian Medical University, Fuzhou, People's Republic of China; ${ }^{5}$ Department of Tuberculosis, Third People's Hospital of Yichang, Yichang, People's Republic of China; ${ }^{6}$ Department of Critical Care, Third People's Hospital of Yichang, Yichang, People's Republic of China

Correspondence: Ze-Hao Zhuang Endoscopy Center, First Affiliated Hospital of Fujian Medical University, 20, Chazhong Road, Taijiang District, Fuzhou, 350005, Fujian Province, People's Republic of China

Tel +86-|3|-7803-24|2

Fax $+89-59187981370$

Email zhuang203@yeah.net
Purpose: COVID-19 is a new infectious disease with global spread. The aim of the present study was to explore possible risk factors and evaluate prognosis in COVID-19 with liver injury. Methods: A retrospective study was conducted on 356 COVID-19 patients in the Third People's Hospital of Yichang, Hubei, China. Clinical characteristics and laboratory tests between patients with and without liver injury were compared, while risk factors of COVID19-related liver injury were analyzed. Univariate and multivariate Cox regression analyses were conducted to identify risk factors of in-hospital death.

Results: Of the patients with liver injury, severe and critical types of COVID-19 comprised $12.43 \%$ and $14.69 \%$, respectively, higher than in patients without liver injury (both $P<0.05$ ). CRP and male sex were independent risk factors for for patients with liver injury, while decreased lymphocyte count (HR 0.024, 95\% CI 0.001-0.821) and elevated monocytes (HR 1.951, 95\% CI 1.040-3.662) and CRP (HR 1.028, 95\% CI 1.010-1.045) were independent risk factors of prognosis of death in COVID-19 patients with liver injury.

Conclusion: Liver injury is a common complication in severe COVID-19 patients. Male sex and elevated CRP were independent risk factors in COVID-19 complicated by liver damage. Liver damage with increased CRP and monocyte count and decreased lymphocyte count may imply a poor prognosis.

Keywords: COVID-19, liver injury, risk factors, prognosis

\section{Introduction}

COVID-19 - infection with severe acute respiratory SARS-CoV2 - was first diagnosed in Wuhan, Hubei province in China., ${ }^{1,2}$ The main manifestations of COVID-19 include fever, dry cough, fatigue, diarrhea, and breathing difficulty, even respiratory distress syndrome in severe cases. It has been demonstrated that SARS-CoV2 can be spread from person to person via airborne droplets and close contact. $^{3}$ COVID-19 is a rapidly increasing public health emergency of international propotions and highly contagious. Recent studies have suggested that COVID-19 patients are predisposed to liver injury, especially severe cases. ${ }^{4-6}$ However, very few studies have been conducted to evaluate the outcome of patients with liver damage. In this study, we retrospectively investigated changes in different types of SARS-CoV2-infected patients, compared clinical characteristics and laboratory test results between normal liver function and liver dysfunction, and analyzed possible risk factors and prognosis of COVID-19 complicated by liver injury. 


\section{Methods}

\section{Subjects}

From January 17 to February 26, 2020, a total of 356 participants referred from the Third People's Hospital of Yichang, Hubei province were consecutively invited to take part in this retrospective study. All patients were confirmed cases of COVID-19 on chest computed tomography (CT) or real-time polymerase chain reaction, according to World Health Organization interim guidance. ${ }^{7}$ Subjects who had a disease history of alcoholic liver disease, viral hepatitis, autoimmune liver disease, liver malignancy, or other known chronic liver disease were excluded. Written informed consent was obtained from all subjects enrolled, and the study was approved by the Ethics Committee of the Third People's Hospital of Yichang. All procedures performed were in accordance with the ethical standards of the institutional research committee and the 1964 Declaration of Helsinki and its later amendments or comparable ethical standards.

\section{Experimental Grouping}

Based on the Chinese diagnosis and treatment program for SARS-CoV2 (version 6), ${ }^{8}$ subjects were divided into mild/ moderate, severe, and critical groups. The mild/moderate group compriseed patients whose manifestations were slight with no signs of pneumonia found on radiography and patients presenting clinical symptoms, such as dry cough, fever, and signs of pneumonia on CT imaging. For the severe group. patients met any of dyspnea (breathing frequency $\geq 30$ times/ minute), resting oxygen saturation $\leq 93 \%$ and arterial partial pressure of oxygen/oxygen concentration $\leq 300 \mathrm{mmHg}$ (1 $\mathrm{mmHg}=0.133 \mathrm{kPa}$ ). In the critical group, patients were up to at least one of the following conditions: respiratory failure and mechanical ventilation, shock, or intensive care-unit monitoring and treatment for multiorgan failure. If liver-function tests met any of the conditions of alanine transaminase (ALT) $>40 \mathrm{U} / \mathrm{L}$, aspartate aminotransferase (AST) $>40 \mathrm{U} / \mathrm{L}$, or total bilirubin (TBil) $>20.4 \mu \mathrm{mol} / \mathrm{L}$, they were defined as having liver injury.

\section{Data Collection}

Demographic features, clinical symptoms, laboratory examination results, medical history, and short-term prognosis were collected. Patients were followed up to April 10, 2020. At the end of the study, all patients were discharged. Demographic features were age, sex, and epidemiological history. Clinical characteristics included fever, dry cough, expectoration, fatigue, diarrhea, headache, dyspnea, and hemorrhage. Laboratory examinations comprising routine blood, CRP, procalcitonin (Pct), liver function, kidney function, D-dimer, and coagulation function were conducted. Medical history included diabetes, chronic liver disease, hypertension, coronary heart disease, cerebrovascular disease, chronic obstructive pulmonary disease, and autoimmune diseases. Duration from symptom onset to hospitalization SARS-CoV2 infection diagnosis, length of hospital stay, and outcomes of all patients were also recorded.

\section{Statistical Analysis}

Categorical variables are described as frequency and percentages, and continuous variables as means, medians, and IQR values. Means for continuous variables were compared using independent-group $t$-tests when data were normally distributed, and otherwise the Mann-Whitney test was used. Categorical variables were compared using $\chi^{2}$. Bivariate logistic regression analysis was used to identify risk factors of patients with liver damage. Univariate and multivariate Cox regression analyses were used to identify risk factors of in-hospital death of liver injury in these patients. Kaplan-Meier survival curves were constructed to compare survival rate for COVID-19 patients with and without liver injury by log-rank test. All statistical analyses were performed using SPSS 25.0. Twotailed $P<0.05$ was considered statistically significant.

\section{Results}

\section{Presenting Characteristics}

We enrolled 356 patients with confirmed COVID-19 from January 17 to February 26, 2020 in Yichang. The average age of patients was 52.11 17.90 years, and 193 (54.21\%) were male. Of all patients, 109 (30.62\%) had one or more coexisting chronic medical conditions. Hypertension (81, $22.75 \%$ ), diabetes $(38,10.67 \%)$, cardiovascular disease $(19,5.34 \%)$, and cerebrovascular disease $(13,3.65 \%)$ were the most common coexisting conditions. Common symptoms at onset of illness included fever $(278,78.09 \%)$, dry cough $(262,73.60 \%)$, expectoration $(161,45.22 \%)$, and fatigue (125 (35.11\%). Median time from admission to first negative result on pharyngeal swab was 12 days. Median hospital stay was about 23 days (Table 1).

Laboratory measures were recorded on the day of hospital admission for all patients, then they were divided into mild/moderate, severe, and critical groups. There were 
Table I Baseline characteristics of patients with COVID-19

\begin{tabular}{|c|c|c|c|c|c|}
\hline & Total $(n=356)$ & Mild/moderate $(n=291)$ & Severe $(n=32)$ & Critical $(n=33)$ & $P$-value \\
\hline \multicolumn{6}{|c|}{ Clinical and epidemiological characteristics } \\
\hline Female, n (\%) & $163(45.79)$ & $138(47.42)$ & $14(43.75)$ & II (33.33) & 0.165 \\
\hline Male, n (\%) & $193(54.21)$ & $153(52.58)$ & $18(56.25)$ & $22(66.67)$ & \\
\hline Age (years) & $52.11 \pm 17.90$ & $49.78 \pm 17.70$ & $62.22 \pm 14.68$ & $62.85 \pm 15.29$ & $<0.001$ \\
\hline \multicolumn{6}{|l|}{ Comorbidities, n (\%) } \\
\hline Hypertension & $81(22.75)$ & $51(17.53)$ & II (34.38) & $19(57.58)$ & $<0.001$ \\
\hline Diabetes & $38(10.67)$ & $19(6.53)$ & $8(25)$ & II (33.33) & $<0.001$ \\
\hline Cardiovascular disease & $19(5.34)$ & $6(2.06)$ & $5(15.63)$ & $8(24.24)$ & $<0.001$ \\
\hline Cerebrovascular disease & $13(3.65)$ & $4(1.37)$ & $5(15.63)$ & $4(12.12)$ & $<0.001$ \\
\hline COPD & $9(2.53)$ & $4(1.37)$ & $2(6.25)$ & $3(9.09)$ & 0.003 \\
\hline Malignancy & $4(1.12)$ & I (0.34) & I (3.13) & $2(6.06)$ & 0.002 \\
\hline \multicolumn{6}{|l|}{ Symptoms, n (\%) } \\
\hline Fever & 278 (78.09) & $218(74.91)$ & $29(90.63)$ & 31 (93.94) & 0.002 \\
\hline Dry cough & $262(73.60)$ & 206 (70.79) & $25(78.13)$ & 31 (93.94) & 0.008 \\
\hline Expectoration & 161 (45.22) & 114 (39.18) & $21(65.63)$ & 26 (78.79) & $<0.001$ \\
\hline Dyspnea & $43(12.08)$ & II (3.78) & $10(31.25)$ & $22(66.67)$ & $<0.001$ \\
\hline Headache & $15(4.21)$ & $10(3.44)$ & $2(6.25)$ & $3(9.09)$ & 0.112 \\
\hline Fatigue & $125(35.11)$ & $92(31.62)$ & $16(50.00)$ & $17(5 \mid .52)$ & 0.004 \\
\hline Diarrhea & $13(3.65)$ & $12(4.12)$ & 0 & I (3.03) & 0.351 \\
\hline Hemorrhage & $7(1.97)$ & $3(1.03)$ & I (3.13) & $3(9.09)$ & 0.004 \\
\hline Length of stay (days) & $23(18-30.75)$ & $22.5(18-30.25)$ & $35(33.5-37.75)$ & $18(14-39.5)$ & $<0.001$ \\
\hline \multicolumn{6}{|c|}{ Laboratory parameters on admission } \\
\hline Leukocyte count $\left(\times 10^{9} / \mathrm{L}\right)$ & $4.40(3.40-5.70)$ & $4.30(3.20-5.60)$ & $4.25(3.65-7.43)$ & $5.50(4.25-7.80)$ & 0.096 \\
\hline Neutrophil count $\left(\times 10^{9} / \mathrm{L}\right)$ & $2.70(1.94-3.8 I)$ & $2.47(1.78-3.69)$ & $3.10(2.08-6.29)$ & $4.33(2.88-6.94)$ & $<0.001$ \\
\hline Monocyte count $\left(\times 10^{9} / \mathrm{L}\right)$ & $0.30(0.22-0.40)$ & $0.32(0.25-0.4 I)$ & $0.19(0.14-0.37)$ & $0.29(0.22-0.39)$ & 0.031 \\
\hline Lymphocyte count $\left(\times 10^{9} / \mathrm{L}\right)$ & $1.19 \pm 0.53$ & $1.26 \pm 0.52$ & $0.89 \pm 0.43$ & $0.82 \pm 0.39$ & $<0.001$ \\
\hline Albumin $(g / L)$ & $37.50(34.20-41.00)$ & $38.40(35.15-42.60)$ & $35.65(28.25-39.83)$ & $31.20(28.55-33.55)$ & $<0.001$ \\
\hline Globulin (g/L) & $26.44 \pm 4.10$ & $26.07 \pm 3.89$ & $28.07 \pm 5.06$ & $28.16 \pm 4.18$ & 0.002 \\
\hline $\operatorname{ALT}(\mathrm{U} / \mathrm{L})$ & $20(13-33)$ & $21(14-37)$ & $21(17-40)$ & $25(14-35)$ & 0.895 \\
\hline AST (U/L) & $21(16-28)$ & $21(16-28)$ & $28(24-36)$ & $26(22-43)$ & $<0.001$ \\
\hline TBil $(\mu \mathrm{mol} / \mathrm{L})$ & $9.19(6.66-13.78)$ & $9.08(6.23-14.43)$ & $8.16(6.18-9.95)$ & $10.57(8.16-15.76)$ & $0.41 I$ \\
\hline Hemoglobin (g/L) & $121(109-133)$ & $122(112-136)$ & $127(106-136)$ & $113(105-130)$ & 0.009 \\
\hline Platelets $\left(\times 10^{9} / \mathrm{L}\right)$ & $139(110-188)$ & $152(109-184)$ & $107.5(83-130)$ & $150(97-180)$ & 0.026 \\
\hline LDH (U/L) & $207(164-264)$ & $210.5(164-255)$ & $282(22|-3| 9)$ & $332(232-458)$ & $<0.001$ \\
\hline \multicolumn{6}{|c|}{ Peak laboratory parameters } \\
\hline $\operatorname{ALT}(\mathrm{U} / \mathrm{L})$ & $26(20-40)$ & $24(20-38)$ & $51(28-65)$ & $58(43-177)$ & $<0.001$ \\
\hline AST(U/L) & $36(24-6 I)$ & $33(2 \mid-57)$ & $71(37-118)$ & $76(34-3 \mid 2)$ & $<0.001$ \\
\hline PT (seconds) & $10.9(10.5-11.3)$ & $10.6(10.3-11.2)$ & $11.25(10.7-12.2)$ & $11.9(11.0-16.3)$ & $<0.001$ \\
\hline APTT (seconds) & $29.7(26.3-33.7)$ & $28.6(26.0-32.0)$ & $30.6(27.8-34.1)$ & $33.4(28.6-42.9)$ & 0.001 \\
\hline D-dimer (mg/L) & $0.59(0.51-1.30)$ & $0.58(0.50-0.94)$ & $2.74(1.24-12.13)$ & $10.75(4.98-27.85)$ & $<0.001$ \\
\hline Lactate $(\mathrm{mmol} / \mathrm{L})$ & $2.20(1.65-2.95)$ & $1.96(1.57-2.81)$ & $2.28(1.89-3.81)$ & $3.27(2.40-4.97)$ & $<0.001$ \\
\hline CK (U/L) & $63.0(41.0-1 \mid 2.0)$ & $59.5(41.0-120.0)$ & $103.5(76.0-283.5)$ & $213.0(66.0-430.5)$ & $<0.001$ \\
\hline CK-MB (U/L) & $12.1(9.4-16.9)$ & $10.15(8.6-13.6)$ & 15.7 (I2.7-20.9) & $26.3(14.2-62.0)$ & $<0.001$ \\
\hline CRP (mg/L) & $21.4(4.8-51.8)$ & $20.0(4.5-42.3)$ & $68.1(44.2-114.1)$ & $|4| .0(63.3-\mid 82.9)$ & $<0.001$ \\
\hline Pct $(\mathrm{ng} / \mathrm{mL})$ & $0.08(0.05-0.13)$ & $0.08(0.05-0.12)$ & $0.17(0.11-0.52)$ & $1.25(0.16-7.08)$ & $<0.001$ \\
\hline
\end{tabular}

Abbreviations: COPD, chronic obstructive pulmonary disease; ALT, alanine aminotransferase; AST, aspartate aminotransferase; TBil, total bilirubin; PT, prothrombin time; APTT, activated partial thromboplastin time; LDH, lactate dehydrogenase; CK, creatine kinase; CK-MB, MB isoenzyme of creatine kinase; Pct, procalcitonin. 
numerous differences in laboratory findings among the groups. Neutrophil count, albumin, AST, hemoglobin, and lactate dehydrogenase (LDH) on admission in the critical group were significantly higher than the other two, as well as peak ALT, AST, prothrombin time (PT), activated partial thromboplastin time (APTT), D-dimer, lactate, creatine kinase $(\mathrm{CK})$, and $\mathrm{CK}-\mathrm{MB}$ isoenzyme (all $P<0.05)$. Moreover, lymphocyte counts on admission in critical patients were lower than in the mild/moderate and severe groups $(P<0.05$; Table 1$)$.

\section{Clinical Features and Laboratory}

\section{Parameters}

Compared with patients with normal liver function, those with liver damage had more hypertension (28.81\% vs $16.76 \%$ ), diabetes (14.69\% vs $6.70 \%$ ), cardiovascular disease $(8.47 \%$ vs $2.23 \%)$, malignancy $(2.26 \%$ vs 0$)$, and dyspnea (18.08\% vs 6.15\%). However, age showed no significant difference between the groups. More importantly, the rate of severe and critical types was markedly increased in COVID-19 patients with liver damage over those without liver injury $(12.43 \%$ vs $5.59 \%$ and $14.69 \%$ vs $3.91 \%$; both $P<0.05)$, and men $(P<0.05)$. Patients with liver damage stayed in hospital longer than patients without liver injury $(P<0.05$; Table 2$)$.

Comparisons of laboratory parameters between patients with and without liver injury are provided in Table 3. There were higher levels of ALT, AST, TBil, LDH on admission, and lower platelet counts in the liverdamage group than the normal liver-function group (all $P<0.05)$. There were increased peak values of ALT, AST, CRP, Pct, PT, APTT, D-dimer, CK, CK-MB, and lactate in the liver-injury group (all $P<0.05$ ). Leukocyte, lymphocyte, and neutrophils number showed no statistical difference between patients with and without liver injury (all $P>0.05)$.

\section{Risk Factors of COVID-19 Patients with Liver Injury}

Compared with patients with normal liver function, 19 factors for patients with liver injury were identified on univariate analysis, including increased male numbers, hypertension, diabetes, cardiovascular disease, and dyspnea, some on admission parameters (eg, LDH and platelet, leukocyte, neutrophil, lymphocyte, and monocyte counts), some on peak parameters (eg, PT, APTT, D-dimer, lactate, CK, CK-MB, CRP, and Pct). Based
Table 2 Characteristics of COVID-19 patients with and without liver injury

\begin{tabular}{|c|c|c|c|}
\hline & $\begin{array}{l}\text { No liver injury } \\
(n=\mid 79)\end{array}$ & $\begin{array}{l}\text { Liver injury } \\
(n=177)\end{array}$ & $P$-value \\
\hline \multicolumn{4}{|c|}{ Clinical and epidemiological characteristics } \\
\hline $\begin{array}{l}\text { Female, } \mathrm{n}(\%) \\
\text { Male, } \mathrm{n}(\%) \\
\text { Age (years) } \\
\text { Length of stay } \\
\text { (days) }\end{array}$ & $\begin{array}{l}104(58.10) \\
75(41.90) \\
53.5(43.5-66.5) \\
20.5(17-29)\end{array}$ & $\begin{array}{l}59(33.33) \\
118(66.67) \\
59(45-68.5) \\
30(21-37)\end{array}$ & $\begin{array}{l}<0.001 \\
0.072 \\
<0.001\end{array}$ \\
\hline \multicolumn{4}{|c|}{ Clinical type on admission, $n$ (\%) } \\
\hline $\begin{array}{l}\text { Mild } \\
\text { Ordinary } \\
\text { Severe } \\
\text { Critical }\end{array}$ & $\begin{array}{l}5(2.79) \\
157(87.71) \\
10(5.59) \\
7(3.91)\end{array}$ & $\begin{array}{l}I(0.56) \\
128(72.32) \\
22(12.43) \\
26(14.69)\end{array}$ & $<0.001$ \\
\hline \multicolumn{4}{|c|}{ Symptoms, n (\%) } \\
\hline $\begin{array}{l}\text { Fever } \\
\text { Dry cough } \\
\text { Expectoration } \\
\text { Fatigue } \\
\text { Dyspnea } \\
\text { Headache } \\
\text { Diarrhea } \\
\text { Hemorrhage }\end{array}$ & $\begin{array}{l}137(76.54) \\
130(72.63) \\
79(44.13) \\
62(34.64) \\
11(6.15) \\
4(2.23) \\
4(2.23) \\
5(2.79)\end{array}$ & $\begin{array}{l}141(79.66) \\
132(74.58) \\
82(46.33) \\
63(35.59) \\
32(18.08) \\
11(6.21) \\
9(5.08) \\
2(1.13)\end{array}$ & $\begin{array}{l}0.476 \\
0.676 \\
0.678 \\
0.85 \\
0.001 \\
0.062 \\
0.152 \\
0.258\end{array}$ \\
\hline \multicolumn{4}{|c|}{ Comorbidities, n (\%) } \\
\hline $\begin{array}{l}\text { Hypertension } \\
\text { Diabetes } \\
\text { Cardiovascular } \\
\text { disease }\end{array}$ & $\begin{array}{l}30(16.76) \\
12(6.70) \\
4(2.23)\end{array}$ & $\begin{array}{l}51(28.81) \\
26(14.69) \\
15(8.47)\end{array}$ & $\begin{array}{l}0.007 \\
0.015 \\
0.009\end{array}$ \\
\hline $\begin{array}{l}\text { Cerebrovascular } \\
\text { disease } \\
\text { COPD } \\
\text { Malignancy }\end{array}$ & $\begin{array}{l}4(2.29) \\
3(1.68) \\
0\end{array}$ & $\begin{array}{l}9(5.14) \\
6(3.39) \\
4(2.26)\end{array}$ & $\begin{array}{l}0.265 \\
0.303 \\
0.043\end{array}$ \\
\hline
\end{tabular}

Abbreviation: COPD, chronic obstructive pulmonary disease.

on these variables, further multivariate logistic regression analysis using logistic regression was performed, and we found that being male (OR $0.325,95 \% \mathrm{CI}$ 0.119-0.884; $P=0.028$ ) and increased CRP (OR 1.016, 95\% CI 1.002-1.031; $P=0.027$ levels were independent predictors for COVID-19 patients with liver injury (Table 4).

\section{Prognosis of COVID-19 Complicated by Liver Injury}

Univariate Cox analyses for patients with liver damage indicated that 23 variables, including age, smoking, 
Table 3 Laboratory parameters in COVID-19 patients with and without liver injury

\begin{tabular}{|c|c|c|c|}
\hline & $\begin{array}{l}\text { No liver injury } \\
(n=179)\end{array}$ & $\begin{array}{l}\text { Liver injury } \\
(n=I 77)\end{array}$ & $P$-value \\
\hline \multicolumn{4}{|l|}{ On admission } \\
\hline $\begin{array}{l}\text { Leukocyte count } \\
\left(\times 10^{9} / \mathrm{L}\right)\end{array}$ & $4.35(3.28-5.63)$ & $\begin{array}{l}4.50 \\
(3.50-5.90)\end{array}$ & 0.268 \\
\hline $\begin{array}{l}\text { Neutrophil count } \\
\left(\times 10^{9} / \mathrm{L}\right)\end{array}$ & $2.40(1.89-3.76)$ & $\begin{array}{l}2.82 \\
(2.10-4.21)\end{array}$ & 0.086 \\
\hline $\begin{array}{l}\text { Lymphocyte count } \\
\left(\times 10^{9} / \mathrm{L}\right)\end{array}$ & $1.21(0.82-1.52)$ & $\begin{array}{l}I .03 \\
(0.76-\mid .4 I)\end{array}$ & 0.114 \\
\hline Platelets $\left(\times 10^{9} / \mathrm{L}\right)$ & $153(113-186)$ & $132(93-180)$ & 0.025 \\
\hline Hemoglobin $(g / L)$ & $117.73 \pm 14.96$ & $124.64 \pm \mid 7.63$ & 0.063 \\
\hline ALT (U/L) & $15(I I-20)$ & $33(22-48)$ & $<0.001$ \\
\hline AST (U/L) & $20(15-23)$ & $29(22-43)$ & $<0.001$ \\
\hline TBil $(\mu \mathrm{mol} / \mathrm{L})$ & 7.35 (5.68-II.32) & $\begin{array}{l}10.00 \\
(7.61-15.24)\end{array}$ & $<0.001$ \\
\hline Albumin $(g / L)$ & $\begin{array}{l}37.35 \\
(34.35-41.80)\end{array}$ & $\begin{array}{l}36.60 \\
(31.90-40.45)\end{array}$ & 0.481 \\
\hline Globulin (g/L) & $26.36 \pm 4.11$ & $26.52 \pm 4.09$ & 0.445 \\
\hline LDH (U/L) & $204(164-25)$ & $253(184-343)$ & $<0.001$ \\
\hline \multicolumn{4}{|l|}{ Peaks } \\
\hline ALT (U/L) & $26(17-32)$ & $66(47-115)$ & $<0.001$ \\
\hline AST (U/L) & $21(17-25)$ & $50(31-67)$ & $<0.001$ \\
\hline CRP (mg/L) & $19.0(1.8-41.5)$ & $\begin{array}{l}51.3 \\
(26.6-85.6)\end{array}$ & $<0.001$ \\
\hline Pct $(\mathrm{ng} / \mathrm{mL})$ & $0.07(0.05-0.11)$ & $\begin{array}{l}0.13 \\
(0.08-0.30)\end{array}$ & $<0.001$ \\
\hline PT (sec) & $10.6(10.4-11.1)$ & $\begin{array}{l}11.0 \\
(10.6-11.8)\end{array}$ & 0.001 \\
\hline APTT (sec) & $28.1(25.5-32.9)$ & $\begin{array}{l}29.7 \\
(27.2-34.2)\end{array}$ & 0.016 \\
\hline D-dimer (mg/L) & $0.59(0.50-1.28)$ & $\begin{array}{l}\text { I.II } \\
(0.56-5.30)\end{array}$ & $<0.001$ \\
\hline CK (U/L) & $57.0(41.8-84.8)$ & $\begin{array}{l}94 \\
(56.5-235.5)\end{array}$ & $<0.001$ \\
\hline CK-MB(U/L) & $9.6(8.6-13.0)$ & $\begin{array}{l}14.0 \\
(10.7-21.0)\end{array}$ & $<0.001$ \\
\hline Lactate $(\mathrm{mmol} / \mathrm{L})$ & I.9I (I.48-2.67) & $\begin{array}{l}2.42 \\
(1.79-3.77)\end{array}$ & 0.001 \\
\hline
\end{tabular}

Abbreviations: ALT, alanine aminotransferase; AST, aspartate aminotransferase; TBil, total bilirubin; LDH, lactate dehydrogenase; Pct, procalcitonin; PT, prothrombin time; APTT, activated partial thromboplastin time; CK, creatine kinase.

hypertension, chronic pulmonary disease, cardiovascular disease, leukocytes on admission, neutrophils on admission, peak PT, elevated CRP, and elevated Pct, among others, were correlated with fatal outcomes. In addition, multivariate Cox regression analyses showed that decreased lymphocyte count on admission (HR 0.024,
Table 4 Multivariate logistic regression analysis of risk factors for COVID-19 patients with liver injury

\begin{tabular}{|l|l|l|l|}
\hline & OR & 95\% Cl & P-value \\
\hline Male sex & 0.325 & $0.119-0.884$ & 0.028 \\
Hypertension & 1.769 & $0.544-5.754$ & 0.343 \\
Diabetes & 0.499 & $0.104-2.388$ & 0.384 \\
Cardiovascular disease & 0.795 & $0.099-6.355$ & 0.828 \\
Dyspnea & 0.915 & $0.207-4.042$ & 0.906 \\
Platelets on admission & 0.999 & $0.99-1.007$ & 0.749 \\
LDH on admission & 1.004 & $0.997-1.010$ & 0.308 \\
Leukocyte count on admission & 1.208 & $0.162-9.014$ & 0.854 \\
Neutrophil count on admission & 0.746 & $0.090-6.161$ & 0.786 \\
Lymphocyte count on admission & 1.136 & $0.094-13.742$ & 0.92 \\
Monocyte count on admission & 0.705 & $0.133-3.730$ & 0.681 \\
Peak PT & 0.818 & $0.599-1.117$ & 0.206 \\
Peak APTT & 0.978 & $0.920-1.040$ & 0.487 \\
Peak D-dimer & 1.012 & $0.945-1.083$ & 0.74 \\
Peak lactate & 1.168 & $0.857-1.592$ & 0.327 \\
Peak CK & 0.999 & $0.996-1.002$ & 0.528 \\
Peak CK-MB & 1.075 & $0.994-1.163$ & 0.071 \\
Peak CRP & 1.016 & $1.002-1.031$ & 0.027 \\
Peak Pct & 1.102 & $0.797-1.525$ & 0.557 \\
\hline
\end{tabular}

Abbreviations: PT, prothrombin time; APTT, activated partial thromboplastin time; LDH, lactate dehydrogenase; CK, creatine kinase; Pct, procalcitonin.

95\% CI $0.001-0.821 ; P=0.039)$, elevated monocytes on admission (HR 1.951, 95\% CI 1.040-3.662; $P=0.037$ ), and peak CRP (HR 1.028, 95\% CI 1.010-1.045; $P=0.002$ ) were independent risk factors of a prognosis of death complicated by liver injury (Table 5).

Kaplan-Meier survival curves showed that cases with liver dysfunction had significantly poorer survival than cases without liver injury, as determined by the log-rank test $\left(\chi^{2}=15.801, P<0.01\right.$; Figure 1). Fifteen (8.4\%) patients with liver injury died, while no patients without liver injury died. Of the 15 who died, the leading cause of death was acute respiratory distress syndrome (13), heart failure (1), and acute kidney failure (1).

\section{Discussion}

In this study, we found that half the COVID-19 patients had abnormal liver function. Elevation of inflammatory markers, liver injury, and coagulation disorders were more frequent in critical patients than mild/moderate and severe patients on hospital admission. Furthermore, the results demonstrated that sex and CRP were independently associated with liver injury. In addition, elevated levels of CRP and monocytes and decreased lymphocyte count were independent risk factors of a fatal prognosis in COVID-19 with liver injury. 
Table 5 Cox regression analyses of risk factors of in-hospital death of COVID-19 patients with liver injury

\begin{tabular}{|c|c|c|c|c|c|c|}
\hline & \multicolumn{3}{|c|}{ Univariate } & \multicolumn{3}{|c|}{ Multivariate } \\
\hline & HR & $95 \% \mathrm{Cl}$ & $P$-value & HR & $95 \% \mathrm{Cl}$ & P-value \\
\hline Age & 1.079 & $1.041-1.119$ & $<0.001$ & - & - & - \\
\hline Smoking & 3.838 & $1.311-11.234$ & 0.014 & - & - & - \\
\hline Hypertension & 13.738 & $4.370-43.190$ & $<0.001$ & - & - & - \\
\hline Diabetes & 3.848 & $1.225-12.088$ & 0.021 & - & - & - \\
\hline Cardiovascular disease & 7.231 & $2.299-22.744$ & 0.001 & - & - & - \\
\hline COPD & 7.685 & $2.164-27.285$ & 0.002 & - & - & - \\
\hline Oxygenation index on admission & 0.993 & $0.988-0.998$ & 0.011 & - & - & - \\
\hline Leukocyte count on admission & 1.138 & $|.004-1.29|$ & 0.044 & - & - & - \\
\hline Neutrophil count on admission & 1.218 & $1.100-1.349$ & $<0.001$ & - & - & - \\
\hline Lymphocyte count on admission & 0.009 & $0.00 I-0.058$ & $<0.001$ & 0.024 & $0.00 \mathrm{I}-0.82 \mathrm{I}$ & 0.039 \\
\hline Monocyte count on admission & 1.875 & $\mid .25 \mathrm{I}-2.8 \mathrm{II}$ & 0.002 & 1.951 & $1.040-3.662$ & 0.037 \\
\hline Hemoglobin on admission & 0.959 & $0.933-0.987$ & 0.004 & - & - & - \\
\hline Albumin on admission & 0.891 & $0.850-0.935$ & $<0.001$ & - & - & - \\
\hline LDH on admission & 1.003 & $1.001-1.004$ & $<0.001$ & - & - & - \\
\hline Peak ALT & 1.002 & $1.001-1.003$ & $<0.001$ & - & - & - \\
\hline Peak AST & 1.002 & $1.001-1.003$ & $<0.001$ & - & - & - \\
\hline Peak PT & 1.162 & $1.103-1.225$ & $<0.001$ & - & - & - \\
\hline Peak APTT & 1.031 & $1.018-1.043$ & $<0.001$ & - & - & - \\
\hline Peak D-dimer & 1.032 & $1.022-1.043$ & $<0.001$ & - & - & - \\
\hline Peak CK & 1.003 & $1.002-1.004$ & $<0.001$ & - & - & - \\
\hline Peak CK-MB & 1.004 & $1.00 \mathrm{I}-1.007$ & 0.012 & - & - & - \\
\hline Peak CRP & 1.027 & $1.018-1.035$ & $<0.001$ & 1.028 & $1.010-1.045$ & 0.002 \\
\hline Peak Pct & 1.094 & $1.051-1.138$ & $<0.001$ & - & - & - \\
\hline
\end{tabular}

Abbreviations: COPD, chronic obstructive pulmonary disease; ALT, alanine aminotransferase; AST, aspartate aminotransferase; PT, prothrombin time; APTT, activated partial thromboplastin time; LDH, lactate dehydrogenase; CK, creatine kinase; Pct, procalcitonin.

Elders and comorbidities, such as hypertension, diabetes, and cardiovascular disease, were more prevalent in the severe and critical groups than the mild/moderate group. In general, elders are more prone to chronic disease, such as hypertension and diabetes. It has been proved that elderly patients have increased severity and fatality in COVID-19. ${ }^{9}$ Therefore, this indicates that comorbidities may be associated with severity of COVID-19 disease.

Recent studies on COVID-19 evaluating the association between liver injury and COVID-19 have shown that patients were at high risk of liver damage, due mainly to raised AST/ATP with slightly elevated Bil. A study on 1,100 COVID-19 patients showed elevated ALT and AST $(>40 \mathrm{IU} / \mathrm{L})$ in $39.4 \%$ and $28.1 \%$ of severe individuals, respectively, and prevalence of increased ALT and AST $19.8 \%$ and $18.2 \%$ among patients without severe disease, respectively. ${ }^{10} \mathrm{~A}$ meta-analysis proved that there was a relationship between liver injury and severity and mortality of patients with COVID-19. ${ }^{11}$ However, Wang et $\mathrm{al}^{9}$ reported there were no differences in ALT between survival and death, and argued that although liver injury was common in patients with infectious SARS-Cov-2, abnormal liver functioning may not a notable symptom of COVID-19 disease. Our study found that AST on admission and at its peak was higher in critical patients than the other two groups, suggesting that AST was associated with disease severity; however, ALT on admission showed no difference among the three groups. The results indicated that AST appears to more sensitive than ALT in predicting COVID-19-complicated liver dysfunction. Similarly, a recent report revealed elevation of AST was more frequent than elevated ALT in ICU patients on admission. ${ }^{12}$ It is noteworthy that AST and ALT are distributed in cardiac, muscle, and other tissue types. Whether AST can be used as an early indicator of COVID-19 disease needs further investigation.

We found that compared with patients without liver injury, more hypercoagulable states, coagulation disorders, and inflammation were found in patients with liver injury, and critical cases were more likely to suffer from liver damage than mild/moderate and severe cases. In addition, elevation of CRP, suggesting bacterial infection, was an 


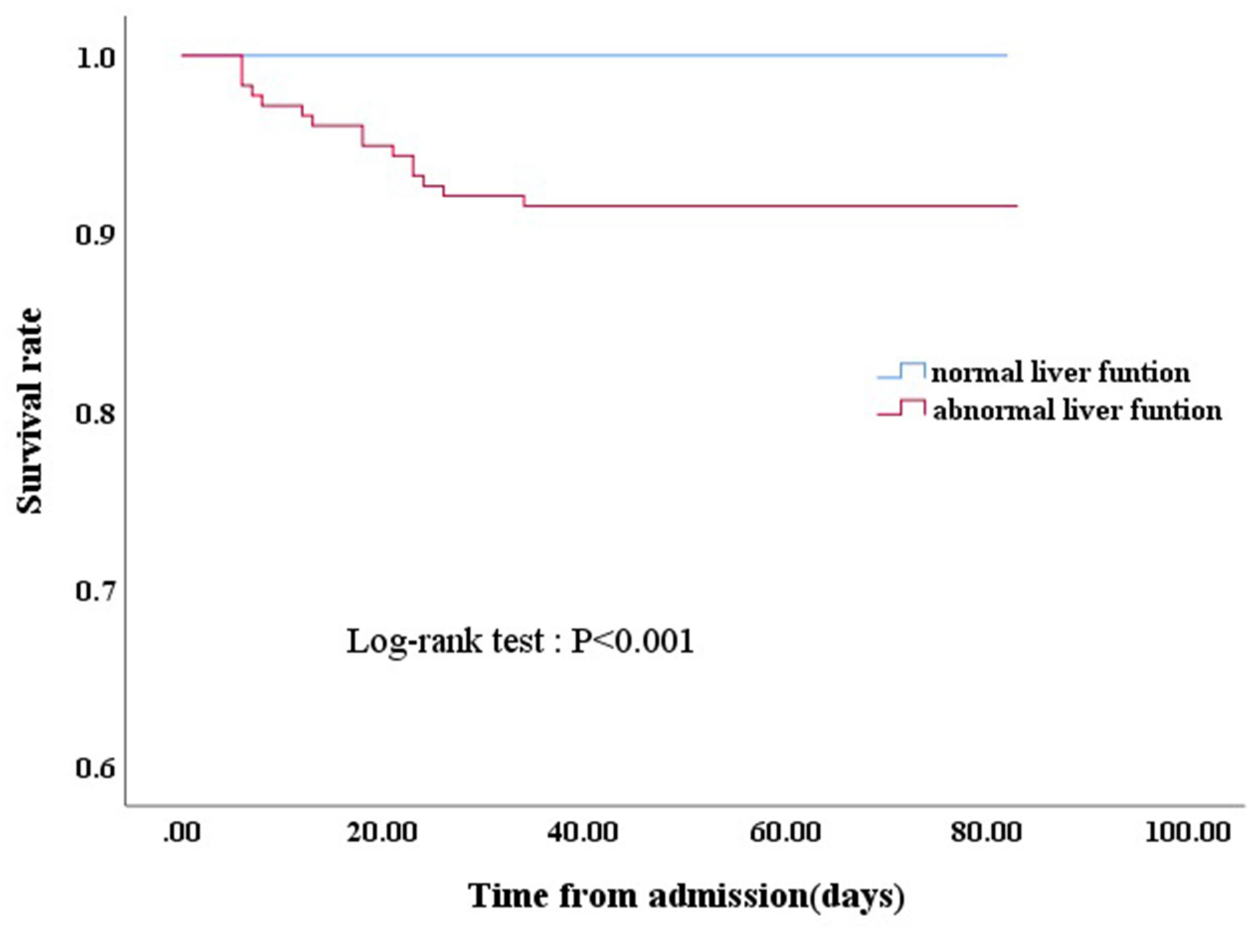

Figure I Kaplan-Meier survival curve for COVID-19 patients with normal liver function and abnormal liver function.

independent risk factor of disease fatality in liver-injury cases. In general, there is a positive correlation between the severity of infection and inflammation, and hepatopathy is often accompanied by high inflammation. Therefore, CRP and Pct, indicators of inflammation, were higher in patents with liver damage in our study than those with normal liver function. Our findings are consistent with previous studies. ${ }^{2,13-15}$ Ali et al ${ }^{16}$ believed CRP levels were related strongly with severity of COVID-19 and may be an appropriate biomarker in assessing conditions of patients combined with clinical symptoms. Luo et al ${ }^{15}$ showed that CRP on admission tended to be a good predictor of adverse outcomes in COVID. Also, findings of inhibition of systemic inflammation by dexamethasone reducing COVID-19-related mortality indicates that there may be a causal association between systemic inflammation and prognosis in COVID-19 patients. ${ }^{17}$ Inflammation storm may contribute to liver injury in COVID-19, with a possible reason being that immunomediated inflammation is harmful to hepatocytes through hypoxia leading to liver dysfunction. Besides, these observations indicate that early secondary bacterial infections may play a vital part in disease progression.

Despite COVID-19 posing a high risk of liver injury in several studies, mechanisms of liver dysfunction during
SARS-CoV2 infection are still unclear. One possible reason may be inflammatory storm, as already mentioned. Another liver-injury mechanism could be related to direct viral attack. It has been demonstrated SARS-CoV2 uses ACE2 as a receptor for invading host cells. ${ }^{18}$ ACE2 is expressed in alveolar type 2 lung cells, bile-duct cells, liver cells, and blood vessels. ${ }^{19}$ In addition, ACE2 expression in bile-duct epithelial cells is much higher than in liver cells, suggesting that the bile duct may be a direct target for SARS-CoV2 injuring the liver. Autopsy analysis of COVID-19 has shown that liver tissue had microvascular steatosis and focal central lobular necrosis with neutrophil infiltration, ${ }^{20}$ suggesting damage induced by SARS-CoV2 infection or drug-induced liver injury. Drug hepatotoxicity may also play a role in liver damage occurring during COVID-19. Our study found that peak ALT and AST was higher in severe and critical patients during hospitalization. Although liver enzymes on hospital admission were in the normal range, they elevated after hospitalization in severe and critical patients. Generally speaking, the short period and regular doses use of these drugs may not cause clinically noticeable liver abnormalities. It is feasible that liver injury might be caused by combinations of systemic inflammation, hypoxic status, direct viral attack, and drug hepatotoxicity in severe and 
critical cases, while for mild and moderate patients, drug side effects possibly plays an important role in liver impairment. This suggests that more attention should be paid to the application of medication that may cause liver damage.

This research also revealed that males were independently predisposed to abnormal liver enzymes. More recently, a study ${ }^{21}$ comprising 72,314 patients identified a higher proportion of males in a COVID-19 group with hepatic dysfunction. Similarly, Xie et $\mathrm{al}^{22}$ demonstrated that male sex was correlated with elevation of liver enzymes. A meta-analysis revealed that sex played role in COVID-19 infection, with men showing higher morbidity, incidence of severe disease, and mortality than women. ${ }^{23}$ A possible explanation for males being susceptible to liver damage is that they have a higher percentage of ACE2 expression than women. ${ }^{24}$ Also, androgen receptors, which may directly regulate the ACE2 expression are positively correlated with ACE2. However, our study found that sex was not a risk factor of death in COVID-19 patients with liver injury on multivariate Cox regression analysis.

We study found that the liver-injury group had shorter median survival following hospital admission than those without liver injury. Meanwhile, increased CRP and monocytes on admission and decreased lymphocyte counts were independent risk factors of mortality for COVID-19 with liver injury on multivariate Cox regression analyses. In line with our study, recent studies have found that the decline in lymphocyte count was related to poor prognosis in the progression of COVID-19. ${ }^{25,26}$ Sun et $\mathrm{al}^{27}$ posited that decreased lymphocyte count on admission was a risk factor of mortality for patients hospitalized with COVID-19. Lymphocytes and their subsets play a significant part in immune-system function. In patients with SARS-CoV2 infection, increased exhaustion and diminished functional diversity of $\mathrm{T}$ cells may predict severe disease. ${ }^{28}$ Direct viral invasion, immunoimpairment by inflammatory mediators, and lymphocyte recruitment by the pulmonary system and infiltrating into the airway may partly explain lymphopenia in COVID-19. Furthermore, liver injury may due to immune-system dysfunction, further aggravating the decreased lymphocyte count in COVID-19 patients. Therefore, for patients with liver injury, lower lymphocyte counts could mean the probability of death rising. A recent study has confirmed ACE2 expression in bronchial epithelium is upregulated by COPD and negatively related to $\mathrm{FEV}_{1} \%$ predicted, suggesting COPD may promote SARS-CoV2 cellular entry because of increased access to AEC receptors. However, our multivariate analyses showed COPD was not a risk factor of in-hospital death in patients with liver injury. This may be related to low percentages in the severe and critical groups and the few deaths in our study. Further longer-term and larger-sample studies are required to confirm risk factors of fatal outcomes in COVID-19 with liver dysfunction.

This study has some limitations. Firstly, we used a retrospective design, which cannot show a causative relationship between liver dysfunction and COVID-19. Secondly, all patients were from a single center and some cases did not have a complete history of illness, laboratory testing, or drug treatment. Thirdly, we did not match some confounding factors, such as illness and history of cardiovascular disease. Multivariate analysis was conducted to overcome this problem. In addition, we excluded chronic liver disease and probably neglected underlying liver diseases, such as NAFLD and autoimmune hepatitis, in COVID-19 patients with limited medical resources.

\section{Conclusion}

In conclusion, elevation of liver enzymes is associated with the severity of COVID-19. Male sex and CRP were independent risk factors of COVID-19 complicated by liver injury. Increased CRP and monocytes and decreased lymphocyte counts played important roles in contributing to the mortality of COVID-19 patients with liver injury. From a clinical perspective, attention should be paid to monitoring liver function and indicators of inflammation in COVID-19 patients with high disease severity.

\section{Acknowledgments}

We thank our patients for participating in this study. Zhuang Zehao designed the study and revised the manuscript. Shen Jiaxin did the statistical analysis and drafted the manuscript. Zhang Qiaoxian and Huang Jiaofeng collected data. Chen Gongping made critical comments on the manuscript. Fang Yingying and Cheng Aiguo supervised the study .

\section{Disclosure}

The authors state that they have no conflicts of interest regarding the content of this manuscript. 


\section{References}

1. Zhu N, Zhang D, Wang W, et al. A novel coronavirus from patients with pneumonia in China, 2019. $N$ Engl J Med. 2020;382 (8):727-733. doi:10.1056/NEJMoa2001017

2. Huang $\mathrm{C}$, Wang Y, Li X, et al. Clinical features of patients infected with 2019 novel coronavirus in Wuhan, China. Lancet. 2020;395 (10223):497-506. doi:10.1016/S0140-6736(20)30183-5

3. Li Q, Guan X, Wu P, et al. Early transmission dynamics in Wuhan, China, of novel coronavirus-infected pneumonia. $N$ Engl J Med. 2020;382(13):1199-1207. doi:10.1056/NEJMoa2001316

4. Bangash M, Patel J, Parekh D. COVID-19 and the liver: little cause for concern. Lancet Gastroenterol Hepatol. 2020;5(6):529-530. doi:10.1016/S2468-1253(20)30084-4

5. Zhang C, Shi L, Wang F. Liver injury in COVID-19: management and challenges. Lancet Gastroenterol Hepatol. 2020;5(5):428-430. doi:10.1016/S2468-1253(20)30057-1

6. Chen N, Zhou M, Dong X, et al. Epidemiological and clinical characteristics of 99 cases of 2019 novel coronavirus pneumonia in Wuhan, China: a descriptive study. Lancet. 2020;395 (10223):507-513. doi:10.1016/S0140-6736(20)30211-7

7. World Health Organization. Clinical management of severe acute respiratory infection when novel coronavirus (ncoV) infection is suspected: interim guidance. Available from: https://www.who.int/ publications-detail/clinical-managementof-severe-acute-respiratoryinfection-when-novelcoronavirus-(ncov)-infection-is-suspected. Accessed February 3, 2021.

8. Novel coronavirus pneumonia prevention and control program (6rd ed.). Available from: http://www.nhc.gov.cn/yzygj/s7653p/202002/ 8334a8326dd94d329df351d7da8aefc2.shtml. Accessed February 3, 2021.

9. Wang L, He W, Yu X, et al. Coronavirus disease 2019 in elderly patients: characteristics and prognostic factors based on 4-week follow-up. $J \quad$ Infect. $\quad 2020 ; 80(6): 639-645 . \quad$ doi:10.1016/j. jinf.2020.03.019

10. Guan WJ, Ni ZY, Hu Y, et al. Clinical characteristics of coronavirus disease 2019 in China. $N$ Engl J Med. 2020;382(18):1708-1720. doi:10.1056/NEJMoa2002032

11. Wu ZH, Yang DL. A meta-analysis of the impact of COVID-19 on liver dysfunction. Eur J Med Res. 2020;25(1):54. doi:10.1186/ s40001-020-00454-x

12. Wang D, Hu B, Hu C, et al. Clinical characteristics of 138 hospitalized patients with 2019 novel coronavirus-infected pneumonia in Wuhan, China.. JAMA. 2020;323(11):1061-1069. doi:10.1001/ jama.2020.1585

13. Liu J, Li S, Liu J, et al. Longitudinal characteristics of lymphocyte responses and cytokine profiles in the peripheral blood of SARS-CoV-2 infected patients. EBioMedicine. 2020;55:102763. doi:10.1016/j.ebiom.2020.102763

14. Liu Y, Yang Y, Zhang C, et al. Clinical and biochemical indexes from 2019-nCoV infected patients linked to viral loads and lung injury. Sci China Life Sci. 2020;63(3):364-374. doi:10.1007/s11427-020-1643-8
15. Luo X, Zhou W, Yan X, et al. Prognostic value of C-reactive protein in patients with coronavirus 2019. Clin Infect Dis. 2020;71 (16):2174-2179. doi:10.1093/cid/ciaa641

16. Ali N. Elevated level of C-reactive protein may be an early marker to predict risk for severity of COVID-19. J Med Virol. 2020;92 (11):2409-2411. doi:10.1002/jmv.26097

17. Group RC, Horby P, Lim WS, et al. Dexamethasone in hospitalized patients with covid-19 - preliminary report. N Engl J Med. 2020.

18. Hoffmann M, Kleine-Weber H, Krüger N, Müller M, Drosten C, Pöhlmann S. The novel coronavirus 2019 (2019-nCoV) uses the SARS-coronavirus receptor ACE2 and the cellular protease TMPRSS2 for entry into target cells. bioRxiv. 2020.

19. Chai X, Hu L, Zhang Y, et al. Specific ACE2 expression in cholangiocytes may cause liver damage after 2019-nCoV infection. bioRxiv. 2020.

20. Yao X, Li T, He Z, et al. A pathological report of three COVID-19 cases by minimally invasive autopsies. Zhonghua Bing Li Xue Za Zhi. 2020;49:E009. doi:10.3760/cma.j.cn112151-20200312-00193

21. Novel CP. The epidemiological characteristics of an outbreak of 2019 novel coronavirus diseases (COVID-19) in China. Zhonghua Liu Xing Bing Xue Za Zhi. 2020;41(2):145-151. doi:10.3760/cma.j. issn.0254-6450.2020.02.003

22. Xie H, Zhao J, Lian N, Lin S, Xie Q, Zhuo H. Clinical characteristics of non-ICU hospitalized patients with coronavirus disease 2019 and liver injury: a retrospective study. Liver Int. 2020;40(6):1321-1326. doi:10.1111/liv.14449

23. Wei X, Xiao Y-T, Wang J, et al. Sex differences in severity and mortality among patients with COVID-19: evidence from pooled literature analysis and insights from integrated bioinformatic analysis. arXiv. 2020. Available from: https:/ui.adsabs.harvard.edu/ abs/2020arXiv200313547W. Accessed March 1, 2020.

24. Patel SK, Velkoska E, Burrell LM. Emerging markers in cardiovascular disease: where does angiotensin-converting enzyme 2 fit in? Clin Exp Pharmacol Physiol. 2013;40(8):551-559. doi:10.1111/ 1440-1681.12069

25. Ruan Q, Yang K, Wang W, Jiang L, Song J. Correction to: clinical predictors of mortality due to COVID-19 based on an analysis of data of 150 patients from Wuhan, China. Intensive Care Med. 2020;46 (5):846-848.

26. Yang $\mathrm{X}, \mathrm{Yu} \mathrm{Y}, \mathrm{Xu} \mathrm{J}$, et al. Clinical course and outcomes of critically ill patients with SARS-CoV-2 pneumonia in Wuhan, China: a single-centered, retrospective, observational study. Lancet Respir Med. 2020;8(5):475-481. doi:10.1016/S2213-2600(20)30079-5

27. Sun H, Ning R, Tao Y, et al. Risk factors for mortality in 244 older adults with COVID -19 in Wuhan, China: a Retrospective Study. $J$ Am Geriatr Soc. 2020;68(6). doi:10.1111/jgs.16533

28. Zheng H, Zhang M, Yang C, et al. Elevated exhaustion levels and reduced functional diversity of $\mathrm{T}$ cells in peripheral blood may predict severe progression in COVID-19 patients. Cell Mol Immunol. 2020;17(5):541-543. doi:10.1038/s41423-020-0401-3
Journal of Multidisciplinary Healthcare

\section{Publish your work in this journal}

The Journal of Multidisciplinary Healthcare is an international, peerreviewed open-access journal that aims to represent and publish research in healthcare areas delivered by practitioners of different disciplines. This includes studies and reviews conducted by multidisciplinary teams as well as research which evaluates the results or conduct of such teams or healthcare processes in general. The journal covers a very wide range of areas and welcomes submissions from practitioners at all levels, from all over the world. The manuscript management system is completely online and includes a very quick and fair peer-review system. Visit http://www.dovepress.com/testimonials. php to read real quotes from published authors. 